\title{
RELACIÓN ENTRE LAS ESTRATEGIAS DE AFRONTAMIENTO, ANSIEDAD, DEPRESIÓN Y AUTOESTIMA, EN UN GRUPO DE ADULTOS CON DIAGNÓSTICO DE CÁNCER
}

\author{
RELATIONSHIP BETWEEN COPING STRATEGIES, ANXIETY, DEPRESSION AND \\ SELF-ESTEEM IN A GROUP OF ADULT PATIENTS DIAGNOSED WITH CANCER
Eliana ORTIZ GARZÓN*, LAURA PATriCia MÉNDEZ SALAZAR, JosÉ ANTONIO CAMARGO BARRERO, SANDRA ANDrEa CHAVARro, Gina ISABEl TORO CARDONA, MARÍA BEATriz VERNAZA GUERRERO
UNIVERSIDAD DE SAN BUENAVENTURA, BOGOTÁ - COLOMBIA

FECHA RECEPCIÓN: 22/08/2013・ FECHA ACEPTACIÓN: 25/09/2013

Para citar este artículo: Ortiz, E., Méndez, L., Camargo, J., Chavarro, S., Toro, G., \& Vernaza, M. (2014). Relación entre las estrategias de afrontamiento, ansiedad, depresión y autoestima, en un grupo de adultos con diagnóstico de cáncer. Psychologia: avances de la disciplina, 8(1), 77-83

\begin{abstract}
Resumen
El presente estudio es de tipo descriptivo correlacional, tiene como objetivo identificar la relación existente entre las estrategias de afrontamiento, los niveles de autoestima, ansiedad y depresión en un grupo de 17 pacientes adultos con diagnóstico de cáncer, para lo cual se emplearon la Escala de Autoestima de Rosemberg, la Escala Hospitalaria de Ansiedad y Depresión (HAD) y la Escala de Estrategias de Coping Modificada (EEC-M). Se obtuvo como resultado una correlación significativa entre los niveles de ansiedad y los niveles de autoestima y entre los niveles de ansiedad y las estrategias de afrontamiento de solución de problemas, reevaluación positiva y reacción agresiva. Al igual que una correlación significativa entre los niveles de depresión y las estrategias de afrontamiento de solución de problemas, negación, reacción agresiva y la expresión de la dificultad de afrontamiento. Palabras clave: cáncer, estrategias de afrontamiento, ansiedad, depresión, autoestima.
\end{abstract}

\section{Abstract}

To carry out this project a descriptive correlational design was used in order to identify the existent relationship between coping strategies, self-esteem, anxiety levels and depression in a group of 17 adult patients diagnosed with cancer. The Rosenberg Self-Esteem Scale, The Hospital Anxiety and Depression Scale (HAD) and the modified Coping Strategies Scale (EEC-M) were applied for this purpose. This research showed the following results: A correlation between anxiety and self-esteem levels, anxiety levels and coping strategies for problem solving, positive reappraisal and aggressive reaction. Likewise, a meaningful correlation between depression levels and coping strategies for problem solving, negation, aggressive reaction and the expression of the coping difficulty were observed.

Keywords: cancer, coping strategies, anxiety, depression, self-esteem.

* Magíster en Psicología Clínica y de la Salud. Docente Facultad de Psicología, Universidad de San Buenaventura, Bogotá, Colombia. Correspondencia: carrera 8 H n.o 172-20, Bogotá, Colombia.eortiz@usbbog.edu.co. 


\section{Introducción}

El cáncer es una enfermedad provocada por la proliferación anormal de células, mediante un proceso denominado carcinogénesis, es decir, la capacidad que adquieren algunas células para multiplicarse incontroladamente e invadir otros órganos (Macarulla, Ramos \& Tabernero, 2009).

Según la Organización Mundial de la Salud (2013), el cáncer es una de las principales causas de muerte en el mundo, siendo el de pulmón, estomago, hígado, colon y mama los que se asocian con mayores índices de mortalidad. En Colombia, según el Instituto Nacional de Cancerología para el 2008, se habían reportado un total de 5418 casos nuevos de cáncer, cifra que según el último dato disponible aumentó a 6313 en el año 2010, lo que evidencia un incremento de 895 nuevos casos más, que los reportados en el 2008 (Instituto Nacional de Cancerología, 2013).

En la actualidad, el tratamiento médico del cáncer depende en gran parte de la localización y del grado de avance en el que se encuentra la enfermedad. Existiendo de esta forma diferentes tratamientos como: la cirugía, la quimioterapia y la radioterapia. Existen además otros tratamientos menos conocidos como la terapia fotodinámica, la terapia hormonal y la terapia biológica o inmunoterapia (Ministerio de la Protección Social \& Instituto Nacional de Cancerología, 2004).

Otra disciplina desde la que se aborda el tratamiento del cáncer es la psicooncología, que se ha interesado por el estudio de los factores psicológicos que se asocian con el origen, diagnóstico y desarrollo de la enfermedad. Se destacan dentro de estos factores las estrategias de afrontamiento, la depresión, la ansiedad y la autoestima.

Las estrategias de afrontamiento en personas con cáncer, hacen referencia al proceso de adaptación mediante el cual se utilizan procesos cognitivos y comportamentales con los que intentan cambiar la situación, en cuanto a su función instrumental; así como de ajustar sus emociones orientándose a encontrarle un sentido a la enfermedad, hacerle frente a la impotencia e incertidumbre, a las dificultades para alcanzar objetivos de vida y a los cambios físicos y psicológicos (Cieslak et al., 2013).
De esta manera, la utilización de las diferentes estrategias de afrontamiento en el desarrollo del cáncer, reduce la aparición de problemas en el transcurso de la misma (Robert et al., 2010). Es así como se ha encontrado que el afrontamiento emocional resulta eficaz en el proceso de ajuste a la enfermedad tanto en hombres como en mujeres (Dalnim, Crystal \& Thomas, 2013).

De acuerdo con esto, en un estudio realizado por Gaviria, Vinaccia, Riveros y Quiceno (2007) se evidenció que los pacientes con diferentes diagnósticos de cáncer y que se encuentran en tratamiento médico, utilizan con mayor frecuencia estrategias de afrontamiento tales como la evitación y la reevaluación positiva.

De la misma forma, la espiritualidad/religión ha sido identificada también como una de las estrategias que tiene impacto positivo en el proceso de ajuste a la enfermedad (Megan \& Erin, 2010), teniendo múltiples funciones a largo plazo, tales como: mantenimiento de la autoestima, provee un sentido, significado y propósito a la vida, puede generar mayor estabilidad emocional y facilita sentimientos de esperanza (Thuné-Boyle, Stygall, Keshtgar \& Newman, 2006).

Se ha evidenciado también que la participación en actividades deportivas constituye una forma de hacer frente a la enfermedad y está asociada con mejor salud psicosocial y calidad de vida en población con diagnóstico de cáncer (Belanger, Plotnikoff, Clark, \& Courneya, 2013).

También se ha constituido en foco de interés, el estudio de los niveles de ansiedad, depresión y autoestima en dicha población. La primera, definida como la anticipación de un daño o desgracia futuros, acompañada de un sentimiento de disforia y/o de síntomas somáticos de tensión (Grupo de trabajo, 2008) y la segunda, entendida como la ausencia de afectividad positiva, pérdida de interés o de la capacidad de disfrutar actividades que normalmente eran placenteras, bajo estado de ánimo y la presencia de una serie de síntomas emocionales, cognitivos, físicos y de conducta asociados (García-Herrera, Nogueras, Muñoz \& Morales, 2011).

Según Gaviria et al. (2007) en los pacientes con diagnóstico de cáncer y que se encuentran en tratamiento médico, no se evidencian síntomas clínicos de ansiedad y depresión. Así mismo, en el transcurso de la enfermedad, no se presentan cambios al respecto. 
Como lo muestra un estudio longitudinal, que buscaba evaluar y comparar la calidad de vida y el estado emocional de 21 pacientes oncológicos entrevistados en dos momentos distintos de su enfermedad; en el que no se encontraron diferencias significativas entre los niveles de ansiedad y depresión registrados al inicio y en sus últimos ciclos de quimioterapia (Terol et al., 2000).

Así mismo, los resultados del estudio realizado por Schroevers, Ranchor y Sanderman (2006), muestran que ocho años después no se presentan diferencias entre las personas sanas y los sobrevivientes del cáncer en cuanto a los niveles de ansiedad, depresión, satisfacción con la vida, autoestima, soporte social y satisfacción con el matrimonio.

En concordancia con otros hallazgos, la autoestima, entendida como las actitudes de aprobación respecto a la capacidad y el valor de sí mismo (Vera, Roselló, \& Toro-Alfonso, 2010), es uno de los factores que influye sobre la imagen corporal y el funcionamiento sexual. De esta manera se ha encontrado que se presenta en niveles altos en los pacientes con diagnóstico de cáncer (Júares \& Landero, 2011; Jucá, Cândido, Lavinas, Moreira, \& Carvalho, 2013). Por lo anterior, el objetivo de la presente investigación es identificar la relación entre las estrategias de afrontamiento, los niveles de autoestima y los niveles de ansiedad y depresión en pacientes adultos con diagnóstico de cáncer.

\section{Método}

\section{Diseño}

Se realizó un estudio de tipo descriptivo correlacional con el fin de establecer múltiples asociaciones y relaciones predictivas entre las variables (Hernández, Fernández, \& Baptista, 2006).

\section{Participantes}

Se trabajó con una muestra no probabilística o dirigida compuesta por 17 sujetos voluntarios, pacientes con diagnóstico de cáncer que se encontraban en tratamiento en una institución para el control del cáncer de la ciudad de Bogotá. Participaron 10 mujeres (58.8\%) y 7 hombres (41.2\%) en un rango de edad de los 29 a los
78 años con un promedio de 52.5 años y una desviación típica de 14.53.

\section{Instrumentos}

Escala de Autoestima de Rosemberg. Cuestionario que explora la autoestima entendida como los sentimientos de valía personal y de respeto a sí mismo. Es una escala autoaplicada tipo Likert, donde los participantes marcan con una «X» las alternativas que más los identifica. Consta de 10 afirmaciones de los sentimientos que tiene la persona sobre ella, 5 direccionadas positivamente (ítems 1-5) y 5 negativamente (ítems 6-10). La calificación de las respuestas se da de 1-4 y se asigna el puntaje inverso a las afirmaciones direccionadas negativamente; los valores teóricos fluctúan entre 10 (baja autoestima) y 40 (alta autoestima). Escala traducida y validada en Chile por Rojas-Barahona, Zegers y Föster (2006), tiene una confiabilidad de .754 .

Escala Hospitalaria de Ansiedad y Depresión (HAD). Permite detectar trastornos de ansiedad y depresión en un medio hospitalario no psiquiátrico o en medios de atención primaria. El contenido de la HAD está referido a los aspectos psicológicos de la ansiedad y depresión. La exclusión de síntomas somáticos (insomnio, fatiga, pérdida de apetito, etc.) es especialmente interesante y evita equívocos de atribución cuando se aplica a individuos con algún tipo de enfermedad (Echeburúa, 1996). Fue creada por Zigmond y Snaith (1983), está estructurada como una escala de tipo Likert que va de 0 a 3 y está conformada por 14 ítems, en donde los pacientes tienen que describir los sentimientos que han experimentado durante la última semana. Consta de dos subescalas de 7 ítems intercalados. La subescala de depresión está centrada en el concepto de anhedonia como síntoma nuclear de este cuadro clínico y que diferencia primordialmente la ansiedad de la depresión. Tanto para la puntuación de ansiedad como de depresión se considera de 0 a 7 normal, de 8 a 10 dudoso y de 11 o más problema clínico. El instrumento mostró un alfa de Cronbach de .85 y la confiabilidad por mitades de .8 en un estudio realizado por Rico, Restrepo y Molina (2005).

Escala de Estrategias de Coping Modificada (EEC-M). Evalúa 12 factores o estrategias de afrontamiento: Solución de Problemas, Búsqueda de Apoyo Social, 
Espera, Religión, Evitación Emocional, Búsqueda de Apoyo Profesional, Reacción Agresiva, Evitación Cognitiva, Reevaluación Positiva, Expresión de la Dificultad de Afrontamiento, Negación y Autonomía. Constituida por 69 ítems con opciones de respuesta tipo Likert, en un rango de frecuencia de 1 a 6 desde nunca hasta siempre. El instrumento fue modificado y se identificaron sus propiedades psicométricas para la población colombiana, teniendo como resultado un Alfa de Cronbach de .847 (Londoño et al., 2006).

\section{Procedimiento}

Una vez obtenido el permiso por parte de las directivas de la institución, se realizaron dos visitas para la aplicación de las pruebas. Se explicó el propósito de la investigación así como los aspectos éticos y se realizó la lectura del consentimiento informado a las personas que decidieron participar. Posteriormente, se continuó con la aplicación de los instrumentos donde cada paciente respondió de manera individual en las instalaciones de la institución y cuando lo requerían contaron con la ayuda de los investigadores a cargo, para orientarlos en el diligenciamiento. La duración aproximada de aplicación de los instrumentos fue de 30 a 40 minutos. Los resultados fueron analizados en el paquete estadístico SPSS versión 20 para Windows.

\section{Consideraciones éticas}

Toda la información obtenida de los participantes se manejó bajo estricta confidencialidad y anonimato.

\section{Resultados}

A continuación se presentan a nivel descriptivo las medidas de tendencia central del estudio: la media y la desviación típica (Desv. Típ.), además las puntuaciones mínimas y máximas de los factores evaluados mediante la aplicación de los instrumentos.

Como se observa en la Tabla 1, las estrategias de afrontamiento más utilizadas son la Solución de problemas, la Religión, la Búsqueda de apoyo profesional y la Reevaluación positiva. La ansiedad, la depresión y la autoestima, en promedio, se encuentran en niveles normales.
Tabla 1. Estadísticas descriptivas

\begin{tabular}{lcc}
\hline \hline & Media & Desv. Típ. \\
\hline Solución de problemas & 4,43 &, 79 \\
Apoyo social & 3,86 & 1,24 \\
Espera & 2,79 &, 90 \\
Religión & 4,45 & 1,39 \\
Evitación emocional & 2,96 &, 91 \\
Apoyo profesional & 4,18 &, 97 \\
Reacción agresiva & 1,96 & 1,17 \\
Evitación cognitiva & 3,71 & 1,27 \\
Reevaluación positiva & 4,41 & 1,08 \\
Expresión de la dificultad de & 2,91 &, 98 \\
afrontamiento & & \\
Negación & 3,45 & 1,41 \\
Autonomía & 2,00 &, 86 \\
ansiedad & 6,64 & 5,24 \\
depresión & 3,82 & 3,35 \\
autoestima & 35,41 & 4,71 \\
\hline \hline
\end{tabular}

Posteriormente, se realizó la correlación de las variables estudiadas tomando como variables predictoras: Estrategias de afrontamiento y autoestima; y como variables criterio: Ansiedad y depresión.

Dicho análisis se obtuvo a través de una correlación de Pearson ( $\mathrm{p} \leq .05)$, como se puede observar en la Tabla 3, se halla una correlación significativa entre los niveles de ansiedad y autoestima (.041), estrategia de Solución de problemas (.023), Reevaluación positiva (.040) y Reacción agresiva (.019).

Tabla 3. Correlación de Pearson para ansiedad

\begin{tabular}{llc}
\hline \hline Factores & & Correlación \\
\hline \hline Autoestima & $\begin{array}{l}\text { Correlación de } \\
\text { Pearson }\end{array}$ &,$- 499 *$ \\
& Sig. (Bilateral) &, 041 \\
Solución de problemas & $\begin{array}{l}\text { Correlación de } \\
\text { Pearson } \\
\text { Sig. (Bilateral) }\end{array}$ &,$- 547 *$ \\
&, 023
\end{tabular}

Esta tabla continúa en la siguiente página $\longrightarrow$ 


\begin{tabular}{llc}
\hline \hline Factores & & Correlación \\
\hline \hline Reevaluación positiva & $\begin{array}{l}\text { Correlación de } \\
\text { Pearson }\end{array}$ &,$- 501 *$ \\
& Sig. (Bilateral) &, 040 \\
Reacción agresiva & $\begin{array}{l}\text { Correlación de } \\
\text { Pearson }\end{array}$ &, $562 *$ \\
& Sig. (Bilateral) &, 019 \\
\hline \hline
\end{tabular}

En cuanto a los niveles de depresión, se encuentra una correlación significativa con la estrategia de Solución de problemas (.050), Negación (.020), Reacción agresiva (.002) y Expresión de la dificultad de afrontamiento (.047) (Ver Tabla 4).

Tabla 4. Correlación de Pearson para depresión

\begin{tabular}{llc}
\hline \hline \multicolumn{1}{c}{ Factores } & Correlación \\
\hline $\begin{array}{l}\text { Solución de } \\
\text { problemas }\end{array}$ & $\begin{array}{l}\text { Correlación de } \\
\text { Pearson }\end{array}$ &,$- 482 *$ \\
& $\begin{array}{l}\text { Sig. (Bilateral) } \\
\text { Negación }\end{array}$ &, 050 \\
& $\begin{array}{l}\text { Correlación de } \\
\text { Pearson }\end{array}$ &,$- 559 *$ \\
& $\begin{array}{l}\text { Sig. (Bilateral) } \\
\text { Correlación de }\end{array}$ &, 020 \\
Reacción agresiva & $\begin{array}{l}\text { Pearson } \\
\text { Sig. (Bilateral) }\end{array}$ &, $002 *$ \\
& $\begin{array}{l}\text { Correlación de } \\
\text { Pearson }\end{array}$ &, $489 *$ \\
$\begin{array}{l}\text { la dificultad de } \\
\text { afrontamiento }\end{array}$ & \begin{tabular}{l} 
Sig. (Bilateral) \\
\hline \hline
\end{tabular} &, 047 \\
\hline \hline
\end{tabular}

\section{Discusión}

El objetivo del presente estudio fue identificar la relación entre las estrategias de afrontamiento, los niveles de autoestima y los niveles de ansiedad y depresión en un grupo de pacientes adultos con diagnóstico de cáncer. Se encontró que las estrategias de afrontamiento más utilizadas por los participantes son: religión, reevaluación positiva, solución de problemas y búsqueda de apoyo profesional. Resultados acordes con los hallados en un estudio de revisión en torno a pacientes con cáncer, donde se reporta que las estrategias de búsqueda de apoyo profesional, acompañado de un alto grado de espiritualidad, fe y religión son las más frecuentes (Vásquez \& Castillas, 2007). Al igual que los resultados de la investigación de Gaviria et al. (2007) quienes reportan que una de las estrategias de afrontamiento más utilizadas en una muestra de pacientes con diferentes diagnósticos de cáncer, es la Reevaluación positiva.

En los resultados del presente estudio, la autoestima, la ansiedad y la depresión se encuentran en niveles normales, lo cual es consistente con los resultados de otras investigaciones al respecto (Fernandes, Alves, Santos, Mota \& Fernandes, 2013; Juárez \& Landero, 2011; Terol et al., 2000). Esto puede deberse a las diferentes estrategias de afrontamiento que se utilizan (Robert et al., 2010), como por ejemplo la religión, que tiene múltiples funciones a largo plazo en el proceso de ajuste a la enfermedad, facilitando el mantenimiento de la autoestima, estabilidad emocional, sentimientos de esperanza y ayudando a proveer un sentido, significado y propósito a la vida (Thuné-Boyle et al., 2006).

Los niveles normales de ansiedad, pueden explicarse además por la correlación negativa hallada con la autoestima, y con la reevaluación positiva que es una de las estrategias de afrontamiento más utilizadas por los participantes.

Así mismo, según Schroevers, Ranchor y Sanderman (2006) la relación existente entre los niveles de ansiedad y la utilización de la estrategia de reevaluación positiva, puede explicar por qué después de 8 años de progresar la enfermedad, y a pesar del aumento en los síntomas físicos, los participantes evaluados, no presentan cambios en los niveles de ansiedad, depresión y autoestima.

En conclusión, se puede afirmar que en la población estudiada, se encontró una relación entre las estrategias de afrontamiento, los niveles de autoestima y los niveles de ansiedad y depresión. De esta manera, se podría concluir que entre más frecuente sea la utilización de estrategias de afrontamiento como la solución de problemas en los pacientes con cáncer, menores serán los niveles de ansiedad y depresión experimentados. Así mismo, entre mayores sean los niveles de autoestima reportados por los pacientes, menores serán los niveles de ansiedad.

Entre las limitaciones del estudio se tiene que los resultados obtenidos no pueden generalizarse a toda la población debido al tamaño de la muestra participante. Y además debe tenerse en cuenta que el instrumento que 
se utilizó para evaluar los niveles de autoestima (Escala de autoestima de Rosemberg) no ha sido validado aún para población colombiana. Por lo tanto, se recomienda para futuras investigaciones ampliar el tamaño de la muestra de tal manera que el análisis estadístico de los datos permita realizar mayores comparaciones y generalizaciones.

\section{Referencias}

Belanger, L., Plotnikoff, R., Clark, A., \& Courneya, K. (2013). Prevalence, correlates and psychosocial outcomes of sport participation in young adult cancer survivors. Psychology of Sport and Exercise, 14, 298-304.

Cieslak, K., Pawlukiewicz, M., Golab, D., Konys, M., Kusnierkiewicz, M., \& Kleka, P. (2013). Styles of coping with stress of cancer in patients treated with radiotherapy and expectations towards medical staff - Practical implications. Reports of Practical Oncology \& Radiotherapy, 18(2), 61-66.

Dalnim, C., Crystal, L., \& Thomas, B. (2013). Emotional approach coping: Gender differences on psychological adjustment in young to middle-aged cancer survivors. Psychology \& Health, 28(8), 1-21.

Echeburúa, E. (1996) Evaluación psicológica de los trastornos de ansiedad. En Manual de Evaluación en Psicología Clínica y Salud. Madrid: Siglo XXI.

Fernandes, M., Alves, P., Santos, M., Mota, E., \& Fernandes, A. (2013). Autoestima de mujeres mastectomizadas - aplicación de la Escala de Rosemberg. Revista da Rede de Enfermagem do Nordeste, 14(1), 101-108.

García-Herrera, J., Nogueras, E., Muñoz, F., \& Morales J. (2011). Guía de Práctica Clínica para el Tratamiento de la Depresión en Atención Primaria. Málaga: Distrito Sanitario Málaga UGC Salud Mental Hospital Regional Universitario «Carlos Haya».

Gaviria, A., Vinaccia, S., Riveros, M., \& Quiceno, J. (2007). Calidad devida relacionada con la salud, afrontamiento del estrés y emociones negativas en pacientes con cáncer en tratamiento quimioterapéutico. Psicología desde el Caribe, 20, 50-75.

Grupo de Trabajo (2008). Guía de Práctica Clínica para el Manejo de Pacientes con Trastornos de Ansiedad en Atención Primaria. Madrid: Plan Nacional para el SNS del MSC.
Hernández, R., Fernández, C., \& Baptista, P. (2006). Metodología de la investigación. México: MacGraw-Hill. Instituto Nacional de Cancerología. (2013). Cáncer en cifras. Bogotá. Recuperado el 28 de marzo del 2013.

Juárez, D., \& Landero, R. (2011). Imagen corporal, funcionamiento sexual, autoestima y optimismo en mujeres con cáncer de mama. Revista Electrónica Nova Scientia, 7(4), 17-34. Recuperado el 10 de mayo del 2013. http://nova_scientia.delasalle.edu. $\mathrm{mx} /$ numero_7/articulos/NovaScientia_07_017.pdf Jucá, M., Cândido, P., Lavinas, M., Moreira, E., \& Carvalho, A. (2013). Autoestima de mulheres mastectomizadas: aplicação da escala de Rosenberg. Revista RENE, 14(1), 101-108.

Londoño, N., Henao, G., Puerta, I., Posada, S., Arango, D., \& Aguirre-Acevedo, D. (2006). Propiedades psicométricas y validación de la Escala de Estrategias de Coping Modificada (EEC-M) en una muestra colombiana. Universitas Psychologica, 5(2), 327- 349.

Macarulla, T., Ramos, F., \& Tabernero, J. (2009). Comprender el cáncer. Barcelona: Editorial Amat. S.l.

Megan, L., \& Erin, O. (2010). Religious/spiritual coping and adjustment in individuals with cancer: unanswered questions, important trends, and future directions. Mental Health, Religion \& Culture, 13(1), 55-65.

Ministerio de la Protección Social e Instituto Nacional de Cancerología. (2004). El Cáncer: Aspectos básicos sobre su biología, clínica, prevención, diagnóstico y tratamiento. Bogotá. Recuperado el 29 de marzo del 2013. www.cancer.gov.co/documentos/ Cartillas/Elcancer.pdf\# page $=29$

Organización Mundial de la Salud. (2013). Cáncer (Nota descriptiva N. ${ }^{\circ} 297$, febrero 2013). Ginebra: WHO media centre. Recuperado el 01 de abril del 2013. www.who.int/mediacentre/factsheets/fs297/es/

Rico, J., Restrepo, M., \& Molina, M. (2005) Adaptación y Validación de la Escala Hospitalaria de Ansiedad y Depresión (HAD) en una muestra de pacientes con cáncer del Instituto Nacional de Cancerología de Colombia. Avances en Medición, 3, 73-86.

Robert, R., Torfinn, G., Roy, B., Olav, D., Arnstein, F., Sophie, D., Olbjorn, K., Oystein, S., Erik W., \& Alv, A. (2010): A study of coping in long-term testicular cancer survivors. Psychology, Health \& Medicine, 15(2), 146-158. 
Schroevers, M., Ranchor, A., \& Sanderman, R. (2006). Adjustment to cancer in the 8 years following diagnosis: A longitudinal study comparing cancer survivors with healthy individuals. Social Science \& Medicine, 63, 598-610.

Terol, M., López-Roig, S., Rodríguez-Marín, J., Angeles, M., Mora, M., Martín-Aragón, M., LeydaMenéndez, J., Neipp, M., \& Lizón, J. (2000). Diferencias en la calidad de vida: un estudio longitudinal de pacientes de cáncer recibiendo tratamiento de quimioterapia. Anales de Psicología, 16(2), 111-122.
Thuné-Boyle, I., Stygall, J., Keshtgar, M., \& Newman, S. (2006). Do Religious/spiritual coping strategies affect illness adjustment in patients with cancer? A systematic review of the literature. Social Science \&Medicine, 63, 151-164.

Vásquez, C., \& Castillas, C. (2007). Emociones positivas y crecimiento postraumático en el cáncer de mama. Psicooncología, 23(4), 388-404.

Vera, B., Roselló, J., \& Toro-Alfonso, J. (2010). Autoestima y juventud puertorriqueña: eficacia de un modelo de intervención para mejorar la autoestima y disminuir los síntomas de depresión. Revista Puertorriqueña de Psicología, 21(1), 35-61. 